\title{
El emprendimiento por necesidad, una ventana hacia el desarrollo de oportunidades de negocios
}

\section{Entrepreneurship by necessity, a window to the development of business opportunities}

Lucía Magdalena Pico Versoza

Universidad Internacional del Ecuador, Ecuador

Autor para correspondencia: lupicove@internacional.edu.ec

Fecha de recepción: 01 de Septiembre de 2016 - Fecha de aceptación: 01 de Noviembre de 2016

\begin{abstract}
Resumen
Este estudio pretende analizar el emprendimiento por necesidad, situación a la que se enfrentan muchos individuos que al incurrir en una aventura empresarial no logran obtener rendimientos crecientes llegando en algunas ocasiones al fracaso del negocio, sin embargo existe la posibilidad de reconocer oportunidades de mercado, las mismas que al ser identificadas oportunamente, crearán una ventana hacia la planificación de estrategias que configuren al emprendedor hacia un posicionamiento y éxito empresarial.
\end{abstract}

Palabras claves: Emprendimiento; necesidad; oportunidades

\begin{abstract}
This study aims to analyze entrepreneurship by necessity, a situation faced by many individuals who, when engaging in an entrepreneurial venture, do not manage to obtain increasing returns, sometimes resulting in a failure of the business, but there is the possibility of recognizing market opportunities. Which, when identified in a timely manner, will create a window for the planning of strategies that will shape the entrepreneur towards positioning and business success.
\end{abstract}

Key words: Entrepreneurship; need; opportunities 


\section{Introducción}

El emprendimiento por necesidad consiste en materializar una idea de negocio en la cual no necesariamente hay potencial crecimiento. Nace de la motivación por crear una solución a una problemática que acontece en un periodo de tiempo determinado y bajo unas circunstancias específicas. Es así que se ejecuta una idea, sin el total conocimiento de su potencial en el mercado, dando paso en algunas ocasiones a una aventura empresarial. Sin embargo, la realidad actual conlleva en su análisis que un emprendimiento bien ejecutado podría convertirse en negocio potencial con muchas oportunidades que lo lleven a posicionarse en el mercado y por ende alcanzar éxito empresarial. Encontrar la clave para realizar esta oportunidad partiendo de la necesidad es la ventana que se pretende descifrar.

En el caso ecuatoriano, se han llevado a cabo varios estudios, uno de ellos, el caso de Global Entrepreneurship Monitor (GEM), en el cual se indica que Ecuador es el segundo país que más emprende en el mundo, alcanzando un índice de actividad emprendedora temprana del $33.6 \%$ a junio del 2016. Esto permite evidenciar que uno de cada tres adultos ha desarrollado un negocio.

En estudios realizados por la Espae Graduate School of Management (2015), se indica que Ecuador obtuvo un índice de Actividad Emprendedora Temprana (TEA) de $32.6 \%$ en el 2014. Esto evidencia que uno de cada tres adultos inició los trámites para establecer un emprendimiento, o poseía un negocio cuya antigüedad no era mayor a los cuatro años. El estudio también permite identificar que $66.4 \%$ de la población encuestada considera al emprendimiento como una buena opción de desarrollo profesional, el $67 \%$ considera que el éxito en el campo de los negocios confiere estatus y respeto, y un $82.9 \%$ está de acuerdo en que el mecanismo utilizado por los medios de comunicación, favorece el emprendimiento en el país.

El emprendimiento se relaciona estrechamente con la creación de empresas, y al hablar de los modelos económicos que llevan a la creación de empresas no podemos dejar de citar lo que indica (Brunet, 2011) entre los cuales distingue el modelo emprendedor de Gartner, el vínculo entre el emprendedor y la teoría de la firma, el emprendedor y la búsqueda de oportunidades, y el modelo teórico del Programa Global Entrepreneurship Monitor (GEM). Todos estos modelos sitúan al hombre como un individuo que actúa permitiéndose y dejándose influenciar por la innovación, clave indispensable en épocas de emprendimiento.

Se considera pertinente citar al autor (Shane, 2003) quien afirma que la creación de empresas está relacionada con el reconocimiento de una oportunidad, siendo esta en muchas ocasiones bajo un criterio subjetivo que permite explotar una idea de negocio y motive al sujeto a buscar los recursos necesarios para llevarla a su ejecución, es decir la materialización de la misma. A esto se suma la importancia de la visión de oportunidad, ésta no debe apagar la planificación necesaria ya que a través de los años se ha evidenciado múltiples negocios que han fracasado precisamente por no haber diseñado previamente un plan de negocios que les permita evaluar la viabilidad de su idea de negocio.

(Acs, 2006) Comenta que la creación de empresas por necesidad refleja la percepción de un individuo de que dicha acciones son la mejor opción de empleo disponible, considerándose 
que esto no tiene incidencia en el desarrollo económico de una nación. Situación contraria se presenta al analizar el emprendimiento por oportunidad, ya que al ser una idea voluntaria y planificada, tiene mayores porcentajes de expectativas ante la productividad y rentabilidad del negocio, situación que incide directamente en el desarrollo productivo de la nación. El autor concluye que es interesante analizar el ratio de emprendimientos por oportunidad en términos de necesidad, como indicador del desarrollo de los países. (Acs, 2006). Contar con una visión de oportunidad es clave para emprender un negocio, ya que tal como lo menciona (Vainrub, 2009) cuando el individuo logra detectar una oportunidad y la combina con entrenamiento formal, la probabilidad de éxito es mucho mayor que sin entrenamiento, tal como generalmente podría llegar a suceder en un país que aún está buscando concienciar la educación como guía para una sociedad del futuro.

A nivel empresarial, las buenas ideas son perdurables y oportunas, y están basadas en productos o servicios que crean o agregan valor a los consumidores (Bygrave, 1997). Es así que, resulta imprescindible que las ideas que se convierten en oportunidades generen valor al cliente rompiendo paradigmas de calidad total.

(Vainrub, 2009) Indica en el gráfico a continuación las características de las oportunidades, las mismas que nacen con una idea que debe ser atractiva, oportuna y perdurable en el tiempo. También hace referencia a que los empresarios experimentados tienden a elegir tres factores del éxito de un negocio, la idea, la oportunidad y los recursos; situación clave para transformarla en oportunidad.

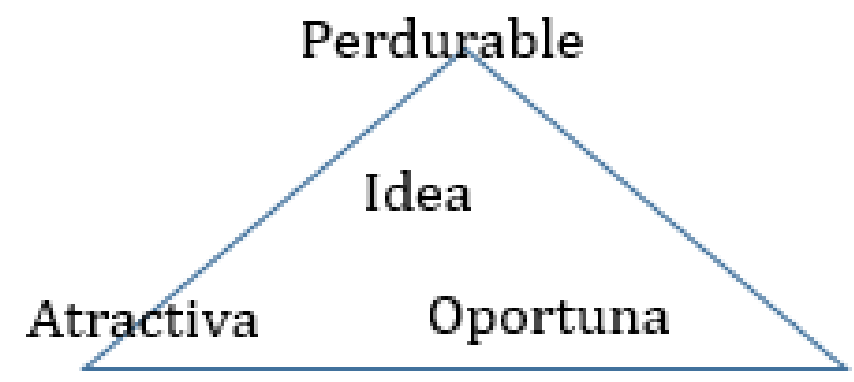

\section{Gráfico No. 1. Reconocimiento de oportunidades Fuente: (Vainrub, 2009)}

La ventana de las oportunidades es un paso fundamental que debe situar al individuo en la búsqueda generar un plan de negocios que evidencia la oportunidad acompañada de los recursos disponibles para su ejecución, a cargo y bajo dirección del emprendedor en todo momento, tal como se muestra a continuación: 


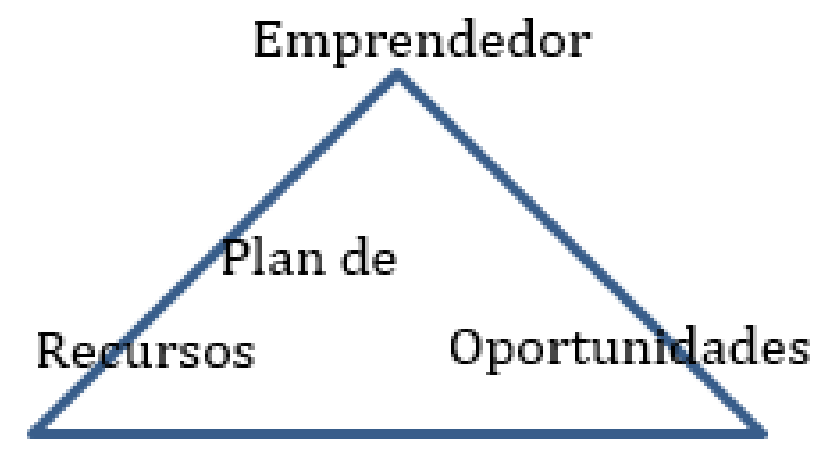

\section{Gráfico No. 2. Factores del éxito empresarial Fuente: (Timmons, 1999}

El emprendimiento está considerado como la capacidad de diseñar una idea, identificar las oportunidades y plasmarlas en un proyecto (Sierra, 2014). Es así que la búsqueda de oportunidad se vuelve imprescindible, siendo el motor que impulsa a viabilizar la idea, generando recursos, opciones y el alcance necesario para lograr el éxito empresarial.

En el Ecuador, el panorama es ligeramente alentador ya que los emprendimientos continúan en crecimiento, sin embargo resulta pertinente analizar los tipos de emprendimientos y bajo qué tipo de características son los que logran perdurar en el tiempo y conseguir el anhelado éxito empresarial. En el año 2015 como se indica en la tabla No. 1, en el Ecuador se registra un $64 \%$ de individuos que percibieron oportunidades, sin embargo solo el $45 \%$ pudo establecer un negocio. Es así que existen individuos que registraron también otras percepciones, miedo al fracaso e intención de emprender.

El emprendimiento mal ejecutado solo se convierte en una idea fugaz que estuvo motivada por la falta de un empleo justo y bien remunerado o simplemente desempleo. La crítica situación que viven los países a nivel mundial y Ecuador no es la excepción ha hecho que los individuos busquen ideas y generen herramientas que permitan viabilizar de forma rápida una idea de negocio, lo cual específicamente en Ecuador es bastante bueno ya que genera un alto índice de emprendimiento. Lo realmente malo es que los emprendimientos por ser generados de forma abrupta y rápida no conllevan una planificación y asesoría para sobrevivir en el tiempo.

Para evaluar la viabilidad de una idea de negocio generalmente se necesita diseñar y construir un plan de negocio que contenga: planificación, ejecución y evaluación; sin embargo muchos individuos por la premura del tiempo no consideran realizarlo con el detalle y precisión que se merece, siendo ésta la principal causa del fracaso.

A partir de lo analizado, resolvemos que el emprendimiento debe ser responsable, resultante de encaminar correctamente las ideas, partiendo de que todo individuo tiene necesidades que deben ser satisfechas. Es así que la propuesta es convertir la necesidad en oportunidad, identificando aspectos claves para iniciar el negocio, logrando sostenibilidad y sustentabilidad, para que a su vez logre maximizar los beneficios tanto de los individuos como del emprendedor. 


\section{Conclusiones}

El emprendimiento se encuentra en crecimiento, sin embargo muchos de ellos no logran consolidarse o llegar al cumplimiento del primer año, debido a la falta de planificación y búsqueda de oportunidades que generen un camino y una meta a perseguir.

Se debe procurar que el emprendimiento esté ligado a situaciones que favorezcan a su desarrollo, esto se conseguirá mediante un estudio de las políticas gubernamentales, disponibilidad de tecnología, educación, apertura del mercado interno y externo.

Los individuos necesitan destacar lo importante que es salir de la zona de confort en la que por distintas situaciones se encuentran inmersos, considerando la opción de generar autoempleo y formas de supervivencia que le permitan además de plasmar una idea de negocio, generar emprendimiento, el cuál inclusive incide directamente en la productividad de la nación, aprovechando cualquier recurso o incentivo que a su vez fortalezca la cultura emprendedora.

La falta de ejecución de un plan de negocios generalmente es el primer motivo del fracaso del emprendimiento en su ejecución, sin embargo se debe procurar analizar la viabilidad a través de un estudio preliminar que incluya el diagnóstico del mercado, estudio técnico y financiero, para de esta forma poder elaborar estrategias que permitan consolidar la idea de negocio y la concesión de cada uno de sus objetivos.

Finalmente, se destaca la importancia de generar una ventana de oportunidades, tarea difícil pero no imposible de viabilizar, rompiendo paradigmas y fortaleciendo cada vez más el emprendimiento llevado a cabo a través de oportunidades, así sea que su nacimiento se haya generado a partir de una necesidad. 


\section{Bibliografía}

Acs, Z. (2006). How Is Entrepreneurship Good for Economic Growth Innovations: Technology, Governance, Globalization, winter 2006, Vol. 1, No. 1, 97-107?

Briasco, I. (2014). El desafío de emprender en el siglo XXI. Madrid, España: Paseo Imperial.

Brunet. (2011). Creación de Empresas. Bogotá: Ediciones de la U.

Bygrave, W. (1997). The portable mba in entrepreneurship. 2da. ed. Nueva York: Wiley.

Caicedo, G. \& Lasio, V. (2016). Emprendimiento en Ecuador: cantidad versus calidad. http://www.espae.espol.edu.ec/images/documentos/publicaciones/publicaciones_medios/ EmpredimientoEcuador.pdf

El Universo (2016) Ecuador es el segundo país que más emprende en el mundo, pero los negocios no se consolidan http://www.eluniverso.com/noticias/2016/06/01/nota/5611422/ecuadores-segundo-pais-que-mas-emprende-mundo-negocios-no-se

ESPAE (2016). Global entrepreurship monitor. http://www.espae.espol.edu.ec/gem2015

Expreso (2014) El autoempleo concentra a los emprendedores http://expreso.ec/historico/elautoempleo-concentra-a-los-emprendedores-HDGR_6215287

Moulden, J. (2009). Los nuevos emprendedores sociales. México: McGraw Hill Interamericana editores, S.A. de C.V.

Shane, S. y. (2003). The individual - opportunity nexus. New York: Springer Publishers.

Sierra, C. P. (2014). Emprendimiento Conceptos y Plan de Negocios. Naucalpan de Juárez, Estado de México: Pearson Educación de México, S.A. de C.V.

Timmons, J. (1999). New venture creation: entrepreneurship for the 21st century. Boston: McGraw-Hill. Universo, D. E. (s.f.).

Vainrub, R. (2009). Una guía para emprendedores. Ciudad de México: Pearson.

White, A. A. (2009). From Confort Zone to Performance Management. 